\title{
Resíduos sólidos urbanos: impactos socioambientais e perspectiva de manejo sustentável com inclusão social
}

\author{
Solid urban waste: socio-environmental impacts \\ and prospects for sustainable management with social inclusion
}

Nelson Gouveia ${ }^{1}$

${ }^{1}$ Departamento de Medicina Preventiva, Faculdade de Medicina, Universidade de São Paulo. Av. Dr. Arnaldo 455. 01246-903 São Paulo SP.ngouveia@usp.br

\begin{abstract}
Strategies to reconcile development with the protection of ecosystems will yet again be discussed at the forthcoming Rio +20 Summit. The management of solid urban waste is an issue which has barely been touched upon in such discussions. Given the institutionalization of the National Solid Waste Policy, this paper seeks to contribute to this debate and to single out alternatives to tackle this issue with an emphasis on social inclusion. For this purpose, specialized scientific literature was consulted as well as information on solid waste management. It is clearly seen that inadequate management of solid waste has immediate impacts on the environment and health, and contributes to climate change. Considering the limitations of the current options for waste disposal, it is essential to minimize the quantities produced by reducing, reusing and recycling. In this context, the role of independent waste gatherers who have been conducting work of great environmental importance is highlighted. Given the vulnerabilities of this population, it is necessary to devise public policies to ensure that waste gathering is a more respected and less risky activity that guarantees an income, so as to move towards more healthy, equitable and sustainable development.
\end{abstract}

Key words Solid waste, Health impacts, Environmental impacts, Recycling, Waste collectors, Social inclusion
Resumo Com a Rio+20 retoma-se a discussão de estratégias para conciliar desenvolvimento com proteção dos ecossistemas. Um tema apenas tangenciado nessas discussões é o gerenciamento dos resíduos sólidos urbanos. Diante da institucionalização da Política Nacional de Resíduos Sólidos, busca-se contribuir para esse debate, bem como apontar caminhos para o enfrentamento dessa questão, privilegiando a inclusão social. Para isso, foram utilizados documentos e informações sobre a gestão de resíduos sólidos, e a literatura científica especializada. Observa-se que o inadequado gerenciamento dos resíduos sólidos gera impactos imediatos no ambiente e na saúde, assim como contribui para mudanças climáticas. Considerando as limitações das opções de destinação final para os resíduos, é imprescindível minimizar as quantidades produzidas por meio da redução, reutilização e reciclagem. Nesse contexto, destaca-se o papel dos catadores, que vêm realizando um trabalho de grande importância ambiental. Dadas as fragilidades desse segmento populacional, é preciso delinear políticas públicas que tornem a atividade de catação mais digna e com menos riscos e que, ao mesmo tempo, garantam renda, para assim caminhar rumo a um desenvolvimento mais saudável, justo e sustentável.

Palavras-chave Resíduos sólidos, Impactos à saúde, Impactos ambientais, Reciclagem, Catadores, Inclusão social 


\section{Introdução}

O debate sobre questões ambientais ganhou grande visibilidade após a Conferência das Nações Unidas sobre Meio Ambiente e Desenvolvimento, Rio-92, quando a discussão sobre os impactos do desenvolvimento nos ecossistemas e na saúde da população se popularizou e conquistou "corações e mentes”. Desde então são buscados mecanismos que atenuem a pressão que o conjunto da sociedade exerce sobre o ambiente de modo a minimizar as alterações no sistema climático planetário, e assim garantir a sobrevivência da vida no planeta. Agora, com a realização da Rio+20, mais uma vez discutem-se estratégias para conciliar o desenvolvimento com a conservação e a proteção de nossos ecossistemas.

Diversas propostas para o enfrentamento das alterações no clima vêm sendo debatidas nessas e em outras conferências similares ${ }^{1}$. O estabelecimento de metas para a emissão dos gases de efeito estufa (GEE), a criação dos Mecanismos de Desenvolvimento Limpo (MDL) e a consequente criação de um mercado de carbono são exemplos de propostas que buscam ao menos tentar mitigar as mudanças climáticas já em curso, por meio de estratégias econômicas.

Um tema de menor destaque nessa discussão, e apenas tangenciado com a implantação dos MDL no Brasil, é o gerenciamento dos resíduos sólidos urbanos. Embora, em termos globais, a queima de combustíveis fósseis (na produção de energia, nos processos industriais e nos transportes) seja a principal fonte de GEE, responsáveis pelas alterações no clima, os resíduos sólidos têm um papel importante nesse cenário, uma vez que também contribuem para a emissão desses gases ${ }^{2}$. O gerenciamento inadequado dos resíduos sólidos urbanos gera diretamente outros impactos importantes, tanto ambientais quanto na saúde da população. Considerandose a tendência de crescimento do problema, os resíduos sólidos vêm ganhando destaque como um grave problema ambiental contemporâneo ${ }^{3}$.

Nesse contexto, busca-se contribuir para a reflexão sobre o impacto da gestão adequada dos resíduos sólidos no meio ambiente, bem como discutir caminhos para o enfrentamento dessa questão, privilegiando ao mesmo tempo a inclusão social. Essa proposta está em consonância com um dos temas centrais da Rio+20, que é a busca do desenvolvimento sustentável com erradicação da pobreza. Para tanto, foram utilizados documentos e informações sobre a gestão de resíduos sólidos disponíveis em diferentes fon- tes, assim como consulta na literatura científica especializada nessa área.

\section{O impacto ambiental}

O desenvolvimento econômico, o crescimento populacional, a urbanização e a revolução tecnológica vêm sendo acompanhados por alterações no estilo de vida e nos modos de produção e consumo da população. Como decorrência direta desses processos, vem ocorrendo um aumento na produção de resíduos sólidos, tanto em quantidade como em diversidade, principalmente nos grandes centros urbanos. Além do acréscimo na quantidade, os resíduos produzidos atualmente passaram a abrigar em sua composição elementos sintéticos e perigosos aos ecossistemas e à saúde humana, em virtude das novas tecnologias incorporadas ao cotidiano ${ }^{4,5}$.

Diariamente, são coletadas no Brasil entre 180 e 250 mil toneladas de resíduos sólidos urbanos $^{6,7}$. A imprecisão nessa estimativa se deve a diferentes metodologias empregadas nos levantamentos realizados e às dificuldades inerentes a essa avaliação. Observa-se ainda que a produção de resíduos está em franca ascensão, com crescimento estimado em $7 \%$ ao ano, valor bastante superior ao $1 \%$ anual observado para o crescimento da população urbana no país recentemente ${ }^{6-10}$. Apesar das grandes diferenças regionais, a produção de resíduos tem crescido em todas as regiões e estados brasileiros. A geração média de resíduos sólidos urbanos é próxima de $1 \mathrm{Kg}$ por habitante/dia no país, padrão já similar ao de alguns países da União Europeia ${ }^{11}$. Entre as populações urbanas mais afluentes o padrão de consumo se equipara ao dos cidadãos norteamericanos, reconhecidamente os maiores produtores per capita de resíduos sólidos urbanos ${ }^{7,12}$.

Entretanto, boa parte dos resíduos produzidos atualmente não possui destinação sanitária e ambientalmente adequada. Embora tenha havido progresso nos últimos vinte anos, os resíduos ainda são depositados em vazadouros a céu aberto, os chamados lixões, em mais da metade dos municípios brasileiros (Tabela 1 ). O percentual de municípios que utilizam aterros controlados, onde os resíduos são apenas cobertos por terra, manteve-se praticamente inalterado entre 2000 e 2008, e houve aumento na destinação para os aterros sanitários, que utilizam tecnologia específica de modo a minimizar os impactos ambientais e os danos ou riscos à saúde humana.

Essa situação é relativamente melhor quando são analisadas as quantidades diárias de resí- 
Tabela 1. Destino final dos resíduos sólidos, por unidades de destino dos resíduos, Brasil - 1989/2008.

\begin{tabular}{cccc}
\hline \multirow{2}{*}{ Ano } & \multicolumn{3}{c}{ Destino final (\%) } \\
\cline { 2 - 4 } & $\begin{array}{c}\text { Vazadouro } \\
\text { a céu aberto }\end{array}$ & $\begin{array}{c}\text { Aterro } \\
\text { controlado }\end{array}$ & $\begin{array}{c}\text { Aterro } \\
\text { sanitário }\end{array}$ \\
\hline 1989 & 88,2 & 9,6 & 1,1 \\
2000 & 72,3 & 22,3 & 17,3 \\
2008 & 50,8 & 22,5 & 27,7 \\
\hline
\end{tabular}

Fonte: IBGE ${ }^{6,8,9}$
Iniciativas para a redução da quantidade de material descartado em aterros, como a coleta seletiva para posterior reciclagem, ainda caminham lentamente. Em 1989 identificou-se a existência de 58 municípios com programas de coleta seletiva de lixo no Brasil. Esse número cresceu para 451 municípios em 2000, e para 994 em 2008, em um universo de 5.564 municípios $^{6-9}$.

O manejo adequado dos resíduos é uma importante estratégia de preservação do meio ambiente, assim como de promoção e proteção da saúde. Uma vez acondicionados em aterros, os resíduos sólidos podem comprometer a qualidade do solo, da água e do ar, por serem fontes de compostos orgânicos voláteis, pesticidas, solventes e metais pesados, entre outros ${ }^{13}$. A decomposição da matéria orgânica presente no lixo resulta na formação de um líquido de cor escura, o chorume, que pode contaminar o solo e as águas superficiais ou subterrâneas pela contaminação do lençol freático. Pode ocorrer também a formação de gases tóxicos, asfixiantes e explosivos que se acumulam no subsolo ou são lançados na atmosfera $^{14}$. Os locais de armazenamento e de disposição final tornam-se ambientes propícios

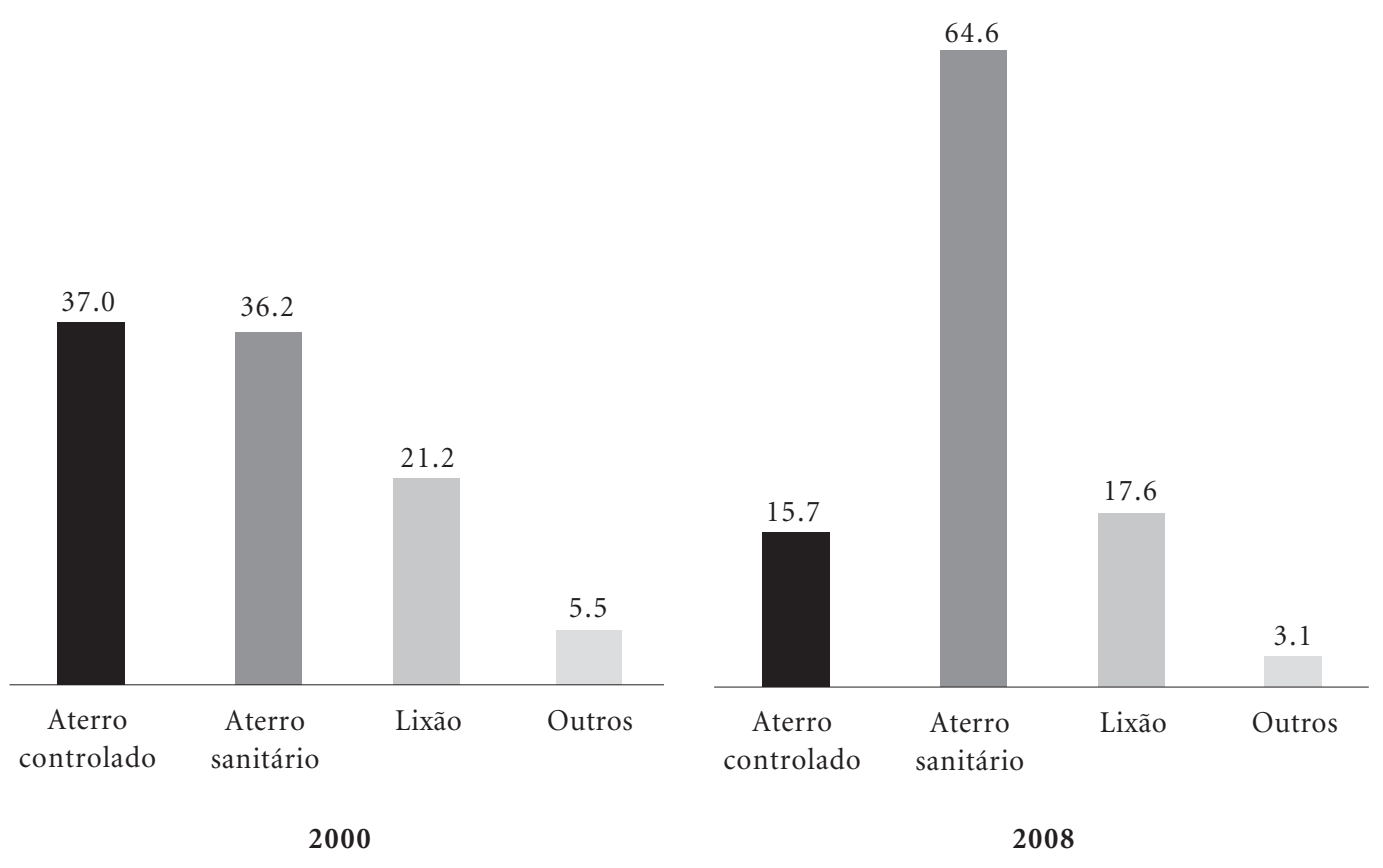

Figura 1. Quantidade diária (\%) de resíduos sólidos, domiciliares e/ou públicos, coletados e/ou recebidos, por unidade de destino final dos resíduos sólidos coletados e/ou recebidos, Brasil 2000 e 2008.

Fonte: $\mathrm{IBGE}^{6,9}$ 
para a proliferação de vetores e de outros agentes transmissores de doenças. Pode haver também a emissão de partículas e outros poluentes atmosféricos, diretamente pela queima de lixo ao ar livre ou pela incineração de dejetos sem o uso de equipamentos de controle adequados. De modo geral, os impactos dessa degradação estendem-se para além das áreas de disposição final dos resíduos, afetando toda a população.

Além desses impactos mais imediatos no ambiente, a disposição de resíduos sólidos pode contribuir de maneira significativa com o processo de mudanças climáticas. A decomposição anaeróbica da matéria orgânica presente nos resíduos gera grandes quantidades de GEE, principalmente o metano $\left(\mathrm{CH}_{4}\right)$, segundo gás em importância dentre os considerados responsáveis pelo aquecimento global ${ }^{15}$. O potencial de emissão de metano aumenta com a melhora das condições de controle dos aterros e da profundidade dos lixões ${ }^{16}$.

Iniciativas para captação do metano gerado em aterros sanitários e sua utilização na geração de energia vêm sendo implantados no âmbito dos projetos de MDL como instrumentos de mitigação de gases de efeito estufa ${ }^{17}$. Entretanto, somente 36 aterros vêm desenvolvendo projetos dessa natureza, em um universo de cerca de 1.500 no Brasil ${ }^{9,17}$.

Segundo o último levantamento feito pelo Painel Intergovernamental de Mudanças Climáticas (IPCC na sigla em inglês), a disposição de resíduos no solo e o tratamento de efluentes são responsáveis por cerca de 3\% das emissões globais de $\mathrm{GEE}^{2}$. No panorama nacional, observase que a contribuição dos resíduos sólidos nas emissões de GEE situa-se em patamar semelhante, com participação de cerca de $2 \%$ no total das emissões. Porém, as variações percentuais acumuladas no período 1990-2005 mostram que as emissões desse setor cresceram a uma taxa de $77 \%$, ou seja, maior do que o crescimento do PIB brasileiro no mesmo período ${ }^{16}$.

Entretanto, é importante salientar que o perfil das emissões brasileiras de GEE é muito especial, influenciado pela matriz energética excepcionalmente limpa e pela grande contribuição das mudanças no uso do solo, em especial pelo desflorestamento e pelas atividades do agronegócio $^{16}$. No nível local, a contribuição dos resíduos adquire grande importância. Por exemplo, inventário realizado no município de São Paulo dá conta de que 23,5\% das emissões de GEE são provenientes da produção de resíduos urbanos ${ }^{18}$.

\section{Os impactos na saúde}

Os vários impactos ambientais decorrentes das diferentes formas de disposição de resíduos sólidos oferecem também riscos importantes à saúde humana. Sua disposição no solo, em lixões ou aterros, por exemplo, constitui uma importante fonte de exposição humana a várias substâncias tóxicas. As principais rotas de exposição a esses contaminantes são a dispersão do solo e do ar contaminado ${ }^{19}$, a lixiviação e a percolagem do chorume $^{20}$. O último pode ocorrer não apenas enquanto o lixão ou o aterro está em funcionamento, mas também depois de sua desativação, uma vez que os produtos orgânicos continuam a degradar. Estudos têm indicado que áreas próximas a aterros apresentam níveis elevados de compostos orgânicos e metais pesados ${ }^{21}$, e que populações residentes nas proximidades desses locais apresentam níveis elevados desses compostos no sangue $^{22}$. Assim, esses depósitos de resíduos sólidos constituem em potenciais fontes de exposição para populações, tendo sido relatado riscos aumentados para diversos tipos de câncer ${ }^{23-25}$, anomalias congênitas ${ }^{26,27}$, baixo peso ao nascer ${ }^{28}$, abortos e mortes neonatai ${ }^{29}$ nessas e em populações vizinhas a esses locais.

Apesar de pouco utilizada no Brasil, a incineração de resíduos também traz riscos à saúde uma vez que produz quantidades variadas de substâncias tóxicas, como gases, partículas, metais pesados, compostos orgânicos, dioxinas e furanos emitidos na atmosfera ${ }^{3}$. A contaminação de populações residentes em áreas próximas a incineradores se dá diretamente (pela inalação de ar contaminado) ou indiretamente (por meio do consumo de água ou alimentos contaminados, ou contato dérmico com solo contaminado $)^{30}$. Vários estudos apontam que a exposição da população à emissão de incineradores está associada a um risco aumentado de alguns tipos de câncer ${ }^{31,32}$, assim como de desfechos indesejados da gravidez, incluindo baixo peso ao nascer e anomalias congênitas ${ }^{30,33}$.

Há ainda os riscos à saúde para os profissionais mais diretamente envolvidos no manejo dos resíduos, como é o caso do pessoal operacional do setor, o qual, em sua maioria, não conta com medidas mínimas de prevenção e segurança ocupacional. Por exemplo, mesmo a compostagem sendo uma destinação ambientalmente mais correta do que a disposição no solo, ela pode gerar impactos à saúde dos trabalhadores desse setor, como alterações na função pulmonar e contaminação bacteriológica do sistema respiratório ${ }^{34-36}$. 
A situação se torna mais crítica para indivíduos que trabalham e vivem da recuperação de materiais do lixo, especialmente os catadores de materiais recicláveis, os quais realizam seu trabalho em condições muito insalubres, geralmente sem equipamentos de proteção, resultando em alta probabilidade de adquirir doenças. Alguns problemas relacionados ao trabalho de reciclagem incluem a exposição a metais e substâncias químicas, a agentes infecciosos como o vírus da hepatite $\mathrm{B}$, doenças respiratórias, osteomusculares e lesões por acidentes ${ }^{4,37}$.

\section{O papel dos catadores nesse cenário}

Os catadores de materiais recicláveis podem ser considerados os grandes protagonistas da indústria de reciclagem no país. Eles detêm posição fundamental na gestão de resíduos sólidos no Brasil, à medida que sua própria existência indica a dificuldade de incluir no gerenciamento desse sistema as atividades de catação, principalmente por problemas de escala de produção combinados a dificuldades logísticas ${ }^{38}$. Esse grupo de trabalhadores vem atuando de maneira informal ou organizada em cooperativas e, mesmo antes da definição de políticas públicas claras para a gestão de resíduos no país, vem realizando um trabalho de grande importância ambiental; contribuindo significativamente para o retorno de diferentes materiais para o ciclo produtivo; gerando economia de energia e de matériaprima, e evitando que diversos materiais sejam destinados a aterros.

A reutilização de resíduos sólidos como insumo nos processos produtivos gera benefícios diretos, tanto na redução da poluição ambiental causada pelos aterros e depósitos de lixo como em benefícios indiretos relacionados à conservação de energia. Em ambas as situações há potencial de diminuição nas emissões de gases responsáveis pelo aquecimento global. Estima-se que, em um cenário ideal de reciclagem, teria sido possível evitar a emissão de 18 a 28 milhões de toneladas de dióxido de carbono no Brasil, no período de 2000 a $2007^{15}$. Portanto, a reciclagem de resíduos sólidos urbanos representa uma importante forma de atenuar os impactos dos gases de efeito estufa, contribuindo em direção a um desenvolvimento mais sustentável.

Todavia, o país ainda apresenta percentuais relativamente baixos de reciclagem. Segundo estimativas para 2006, foram gerados cerca de 50 milhões de toneladas de resíduos sólidos urbanos, dos quais somente $18 \%$ da fração seca (me- tais, papeis, plásticos e vidros) foram devidamente reciclados ${ }^{12}$. Por tipo de material, observa-se que apenas o alumínio atinge taxas de reciclagem próximas de 100\%. Para outros materiais como plásticos e vidros, o percentual ainda está em torno de $40 \%$ do que é produzido ${ }^{39}$.

Para melhorar esses índices, é preciso incentivo à coleta seletiva com adequada separação dos diversos materiais, tanto no momento da geração do resíduo - nesse caso pela população devidamente informada para desempenhar esse papel - quanto nas centrais de triagem. Nesse aspecto, ressalta-se novamente o papel que os catadores de matérias recicláveis vêm desempenhando nessa cadeia produtiva.

Não há levantamentos precisos sobre o número de catadores existentes atualmente, mas algumas previsões apontam para mais de um milhão de trabalhadores espalhados por várias cidades brasileiras. Na Figura 2, visualiza-se o crescimento na última década do número de catadores no Brasil.

Vale destacar que, desde 2002, a atividade de catador foi reconhecida como categoria profissional, registrada na Classificação Brasileira de Ocupação (CBO), sob no 5192-05 como "Catador de Material Reciclável”. Essa nova categoria de trabalhadores exerce a função de coletar, transportar, triar, prensar, armazenar e negociar esses materiais para serem reutilizados ${ }^{38}$. Todavia, para uma adequada inserção desses profissionais no sistema de gerenciamento de resíduos sólidos, é preciso assegurar tanto os aspectos de direito ao trabalho e renda como avaliar as condições de saúde e os riscos aos quais estão expostos.

\section{Manejo sustentável com inclusão social}

O presente artigo buscou desvelar os diversos impactos associados ao inadequado gerenciamento dos resíduos sólidos urbanos, tanto os impactos imediatos ao ambiente e a saúde da população como aqueles mais a longo prazo devidos às alterações climáticas decorrentes das emissões de gases de efeito estufa. A diversidade de substâncias potencialmente tóxicas presentes no lixo urbano, as evidências de contaminação do solo e água subterrânea, e os efeitos já relacionados a essa exposição em populações vizinhas a essas áreas, assim como o potencial de geração de GEE, devem ser considerados no planejamento e execução de políticas de gerenciamento de resíduos.

Nesse sentido, pode-se concluir que é preciso caminhar em direção a uma gestão dos resíduos 


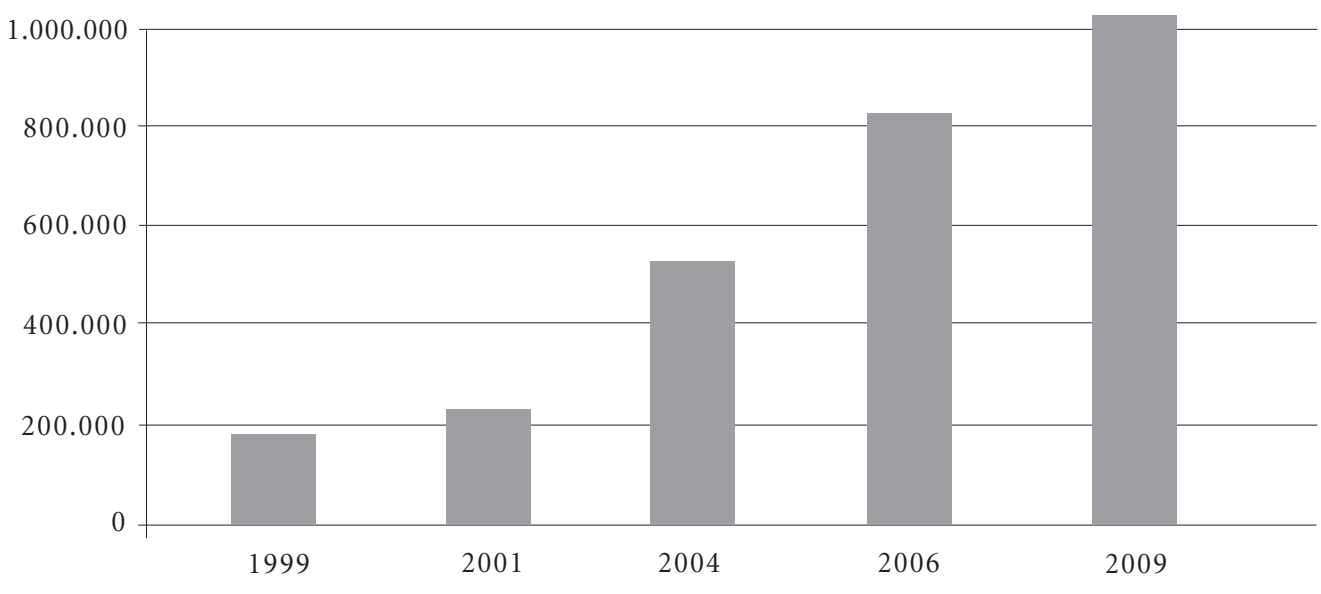

Figura 2. Evolução do número de catadores (autônomos e cooperativados) no Brasil.

Fonte: CEMPRE ${ }^{43}$

sólidos que busque a eliminação de seus impactos negativos no ambiente e na saúde da população. Para tanto, já é possível contar com um marco legal, uma vez que foi sancionada a Política Nacional de Resíduos Sólidos (PNRS) em 2010, com diretrizes para o planejamento e a gestão dos resíduos no país, tais como a obrigatoriedade da preparação de planos municipais de gerenciamento de resíduos, o estabelecimento de prazos para a erradicação dos lixões e a implantação da coleta seletiva ${ }^{40}$.

Contudo, o manejo de resíduos ainda enfrenta sérias limitações, mesmo utilizando tecnologias apropriadas, particularmente em relação à destinação para aterros sanitários e à incineração. A disposição no solo, mesmo que em aterros sanitários com captação de gases e efluentes esbarra no esgotamento de áreas físicas apropriadas para esse fim $^{17}$, principalmente nos grandes centros urbanos, implicando no deslocamento desses resíduos para longas distâncias, com os consequentes transtornos associados ao transporte (poluição, acidentes, etc). Além disso, deve ser considerado o potencial esgotamento dos serviços ecossistêmicos necessários para degradar todo o resíduo depositado ${ }^{41}$.

A incineração, uma das opções para o gerenciamento de resíduos, vem crescendo em muitos países, principalmente em projetos com recuperação energética para produção de eletricidade ${ }^{3}$. Contudo, a segurança da população do entorno em relação aos efluentes lançados ao ar depende de avaliação das tecnologias empregadas, sendo necessário manter o monitoramento de suas emissões e dos possíveis efeitos detectados na saúde ${ }^{42}$. Dessa forma, torna-se imprescindível buscar minimizar a quantidade de resíduos que necessitam de destinação adequada, seguindo a lógica dos três R: redução, reutilização e reciclagem.

A redução e a reutilização, seja essa última diretamente ou através dos processos de compostagem e reciclagem, podem ser incentivadas por meio de ações educativas que visem a atitudes de consumo mais consciente por parte da população. Não obstante as forças do mercado que nos impelem a consumir inconsequentemente, é importante atuar também em direção a novos padrões de consumo. Estes, por sua vez, e novamente por meio das forças do mercado, podem levar a novas formas de produção ambientalmente sustentáveis, podendo ser realizada com menor queima de combustíveis fósseis, principal vilão quando se refere às mudanças climáticas globais.

A reciclagem, como já colocado, precisa ser impulsionada com a implantação da coleta seletiva e triagem dos resíduos. Levando em conta as deficiências em infraestrutura para a realização desse trabalho em grande parte dos municípios brasileiros, deve-se atentar para a PNRS, que propõe a destinação de recursos financeiros para os municípios realizarem trabalho de integração e capacitação de catadores de recicláveis, incentivando a criação e o desenvolvimento de cooperativas ou de outras formas de associação $0^{43}$. Onde já existe uma cadeia informal de reciclagem com- 
posta por esses catadores, deve-se incorporar os seus serviços nos planos de gestão de resíduos sólidos dos municípios.

Entretanto, é preciso considerar o contexto já institucionalizado de vulnerabilidade, precariedade e fragilidade das condições de trabalho dos catadores $^{38}$. Assim, diante da emergente institucionalização da PNRS, e de modo a não reproduzir o efeito perverso da exclusão e da exploração desse segmento de trabalhadores, é importante que se delineiem políticas públicas que articulem aspectos sociais (saúde, segurança do trabalho, autogestão, cidadania, inclusão entre outras), econômicos (geração de renda, redução de custos, mercado entre outros) e técnico-ambientais (qualidade, eficiência entre outras). $\mathrm{O}$ objetivo deve ser tornar a atividade de catação mais digna e com menos riscos e, ao mesmo tempo, garantir a geração de renda e riqueza, fazendo a inclusão social desse segmento importante de trabalhadores, vitais para a mitigação de nossas pegadas ecológicas.

\section{Referências}

1. Viola E. O regime internacional de mudanças climáticas e o Brasil. RBCS 2002; 17(50):25-46.

2. Intergovernmental Panel on Climate Change (IPCC). Climate Change 2007: Synthesis Report. Core Writing Team, Pachauri RK, Reisinger A, editors. Geneva: IPCC; 2007.

3. World Health Organization (WHO). Population health and waste management: scientific data and policy options. Report of a WHO workshop Rome, Italy, 29-30 March 2007. Copenhagen: WHO Regional Office for Europe; 2007.

4. Ferreira JA, Anjos LA. Aspectos de saúde coletiva e ocupacional associados à gestão dos resíduos sólidos municipais. Cad Saude Publica 2001; 17(3):689696.

5. Velloso MP. Processo de Trabalho da Coleta de Lixo Domiciliar na Cidade do Rio de Janeiro: Percepção e Vivência dos Trabalhadores [dissertação]. Rio de Janeiro: Fiocruz; 1995.

6. Instituto Brasileiro de Geografia e Estatística (IBGE). Pesquisa Nacional de Saneamento Básico, PNSB -2008. Rio de Janeiro: IBGE; 2010.

7. Associação Brasileira de Empresas de Limpeza Pública e Resíduos Especiais (Abrelpe). Panorama de Resíduos Sólidos no Brasil- 2010. São Paulo: Abrelpe; 2010 .

8. Instituto Brasileiro de Geografia e Estatística (IBGE). Pesquisa Nacional de Saneamento Básico, PNSB -1991. Rio de Janeiro: IBGE; 1992.

\section{Conclusão}

As decisões que envolvem o gerenciamento de resíduos sólidos urbanos são fundamentalmente decisões sobre saúde pública e requerem, portanto, a integração entre políticas econômicas, sociais e ambientais. O complexo desafio para as grandes cidades na gestão de resíduos sólidos neste início de século pode ser enfrentado pela formulação de políticas públicas que objetivem eliminar os riscos à saúde e ao ambiente, que colaborem na mitigação das mudanças climáticas relacionadas à ação humana e, ao mesmo tempo, garantam a inclusão social efetiva de parcelas significativas da população. Assim, caminharemos rumo a um desenvolvimento mais saudável, em uma perspectiva socialmente justa, ambientalmente sustentável, sanitariamente correta e economicamente solidária.
9. Instituto Brasileiro de Geografia e Estatística (IBGE). Pesquisa Nacional de Saneamento Básico, PNSB -2000. Rio de Janeiro: IBGE; 2002.

10. Associação Brasileira de Empresas de Limpeza Pública e Resíduos Especiais (Abrelpe). Panorama de Resíduos Sólidos no Brasil- 2009. São Paulo: Abrelpe; 2009.

11. European Environment Agency (EEA). Better management of municipal waste will reduce greenhouse gas emissions. European Environment Agency, Briefing 1. Copenhagen: EEA; 2008.

12. Nalini JE. O mercado de reciclagem de lixo no Brasil: entraves ao desenvolvimento [dissertação]. São Paulo; Pontifícia Universidade Católica; 2008.

13. Giusti L. A review of waste management practices and their impact on human health. Waste Manag 2009; 29(8): 2227-2239.

14. Gouveia N, Prado RR. Riscos à saúde em áreas próximas a aterros de resíduos sólidos urbanos. Rev Saude Publica 2010; 44(5):859-866.

15. Pereira AS, Oliveira LB, Reis MM. Emissões de $\mathrm{CO}_{2}$ Evitadas e Outros Benefícios Econômicos e Ambientais Trazidos pela Conservação de Energia Decorrente da Reciclagem de Resíduos Sólidos no Brasil. In: Anais do III Encontro Nacional da Sociedade Brasileira de Economia Ecológica; 1999; Recife [CD-ROM]. 
16. Brasil. Ministério de Ciência e Tecnologia. Inventário Brasileiro das emissões e remoções antrópicas de gases de efeito estufa - Informações Gerais e Valores Preliminares. Brasília: Ministério de Ciência e Tecnologia; 2009.

17. Jacobi P, Besen GR. Gestão de resíduos sólidos em São Paulo: desafios da sustentabilidade. Estudos Avançados 2011; 25(71):135-158.

18. Prefeitura do Município de São Paulo. Inventário de emissões de gases de efeito estufa do Município de São Paulo - Síntese. São Paulo: Prefeitura do Município de São Paulo; 2005.

19. Ward RS, Williams GM, Hills CC. Changes in major and trace components of landfill gas during subsurface migration. Waste Manage Res. 1996; 14(3):243-261.

20. El-Fadel M, Findikakis AN, Leckie JO. Modelling Leachate Generation and Transport in Solid Waste Landfills: Environ Technol. 1997; 18(7):669-686

21. Sissino CLS, Moreira JC. Avaliação da contaminação e poluição ambiental na área de influência do aterro controlado do Morro do Céu, Niterói, Brasil. Cad Saude Publica 1996; 12(4):515-523.

22. Santos Filho E, Souza E, Silva R, Barreto HHC, Inomata ONK, Lemes VRR, Kussumi TA, Rocha SOB. Grau de exposição a praguicidas organoclorados em moradores de aterro a céu aberto. Rev Saude Publica 2003; 37(4):515-522.

23. Goldberg MS, Homnsi AL, Goulet L, Riberdy H. Incidence of cancer among persons living near a municipal solid waste landfill site in Montreal, Quebec. Arch Environ Health. 1995; 50(6):416-424.

24. Goldberg MS. Risks of developing cancer relative to living near a municipal solid waste landfill site in Montreal, Quebec, Canada. Arch Environ Health. 1999; 54(4):291-296.

25. Pukkala E, Pönkä A. Increased incidence of cancer and asthma in houses built on a former dump area. Environ Health Perspect 2001; 109(11):1121-1125.

26. Palmer SR, Dunstan FDJ, Fielder H, Fone DL, Higgs G, Senior ML. Risk of congenital anomalies after the opening of landfill sites. Environ Health Pesp. 2005; 113(10):1362-1365.

27. Elliot P, Richardson S, Abellan JJ, Thomsom A, Hoogh C, Jarup L, Briggs DJ. Geographic density of landfill sites and risk of congenital anomalies in England. Occup Environ Med. 2009; 66(2):81-89.

28. Elliot P, Briggs D, Morris S, Hoogh C, Hurt C, Jensen TK, Maitland I, Richardson S, Wakefield J, Jarup L. Risk of adverse birth outcomes in populations living near landfill sites. BMJ. 2001; 323(7322):363-368.

29. Dummer TJB. Adverse pregnant outcomes near landfill sites in Cumbria, Northwest, England, 195093. Arch of Environ Health 2003; 58(11):692-698

30. Franchini M, Rial M, Buiatti E, Bianchi F. Health effects of exporuse to waste incinerator emissions: a review of epidemiological studies. Ann 1st Super Sanità. 2004; 40(1):101-115

31. Viel JF, Daniau C, Goria S, Fabre P, Crouy-Chanel P, Sauleau EA, Empereur-Bissonnet P. Risk for non Hodgkin's lymphoma in the vicinity of French municipal solid waste incinerators. Environ Health. 2008; 7:51-59.
32. Elliott P, Shaddick G, Kleinschmidt I, Jolley D, Walls P, Beresford J, Grundy C. Cancer incidence near municipal solid waste incinerators in Great Britain. Br J Cancer 1996; 73(5):702-710.

33. Cordier S, Chevrier C, Robert-Gnansia E, Lorente C, Brula P, Hours M. Risk of congenital anomalies in the vicinity of municipal solid waste incinerators. Occup Environ Med. 2004; 61(1):8-15.

34. Athanasiou M, Makrynos G, Dounias G. Respiratory health of municipal solid waste workers. Occup Med 2010; 60(8):618-623.

35. Wouters IM, Hilhorst SKM, Kleppe P, Doekes G, Douwes J, Peretz C, Heederik D. Upper airway inflammation and respiratory symptoms in domestic waste collectors. Occup Environ Med 2002; 59(2): 106-112.

36. Krajewski JA, Tarkowski S, Cyprowski M, Szarapinska-Kwaszewska J, Dudkiewicz B. Occupational exposure to organic dust associeted with mucipal waste collection and manegement. Int J Occup Med Environ Health 2002; 15(3):289-301.

37. Sisinno CL, Oliveira RM. Residuos Sólidos, ambiente e saúde: uma visão muldisciplinar. Rio de Janeiro: Ed. Fiocruz; 2000.

38. Gonçalves-Dias SLF. Catadores: uma perspectiva de sua inserção no campo da indústria de reciclagem [tese]. São Paulo: Universidade de São Paulo; 2009.

39. Pinto-Coelho RM. Reciclagem e Desenvolvimento Sustentável. Belo Horizonte, 2009. [acessado 2012 abr 4]. Disponível em: http://ecologia.icb.ufmg.br/ $\sim$ rpcoelho/Livro_Reciclagem/website/index.htm

40. Brasil. Lei no 12.305 de 02 de Agosto de 2010. Institui a Política Nacional de Resíduos Sólidos; altera a Lei no 9.605 , de 12 de fevereiro de 1998; e dá outras providências. Diário Oficial da União 2010; 3 ago. [acessado $2011 \mathrm{dez}$ 15]. Disponível em: http://www. mncr.org.br/box_2/instrumentos-juridicos/leis-edecretos-federais/Lei\%20\%2012.305-2010\%20Politica \%20de\%20Residuos\%20Solidos.pdf/view

41. Holzman DC. Accounting for nature's benefits. The dollar value of ecosystem services. Environ Health Perspect 2012; 120:153-157

42. Gouveia N, Prado RR. Análise espacial dos riscos à saúde associados à incineração de resíduos sólidos: avaliação preliminar. Rev Bras Epidemiol 2010; 13(1):3-10.

43. Compromisso empresarial para a reciclagem (CEMPRE). Política Nacional de Resíduos Sólidos: a lei na prática. São Paulo: CEMPRE; 2011. [acessado 2011 dez 15]. Disponível em: http://www.cempre.org.br/ download/pnrs_leinapratica.pdf

Artigo apresentado em 17/04/2012

Versão final aprovada em 26/04/2012 